\title{
¿Distributed Sensing of Wind Direction Using Fiber-Optic Cables
}

\author{
ANitA Freundorfer, ${ }^{a}$ KARL LAPO, ${ }^{\mathrm{a}, \mathrm{b}}$ JOHANn SCHNEIDER, ${ }^{\mathrm{a}}$ AND CHRISTOPH K. ThOMAS ${ }^{\mathrm{a}, \mathrm{b}}$

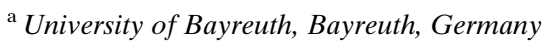 \\ ${ }^{\mathrm{b}}$ Bayreuth Center of Ecology and Environmental Research, Bayreuth, Germany
}

(Manuscript received 26 February 2021, in final form 12 July 2021)

\begin{abstract}
In the atmospheric boundary layer, phenomena exist with challenging properties such as spatial heterogeneity, particularly during stable weak wind situations. Studying spatially heterogeneous features requires spatially distributed measurements on fine spatial and temporal scales. Fiber-optic distributed sensing (FODS) can provide spatially distributed measurements, simultaneously offering a spatial resolution on the order of decimeters and a temporal resolution on the order of seconds. While FODS has already been deployed to study various variables, FODS wind direction sensing has only been demonstrated in idealized wind tunnel experiments. We present the first distributed observations of FODS wind directions from field data. The wind direction sensing is accomplished by using pairs of actively heated fiber-optic cables with cone-shaped microstructures attached to them. Here we present three different methods of calculating wind directions from the FODS measurements, two based on using combined wind speed and direction information and one deriving wind direction independently from FODS wind speed. For each approach, the effective temporal and spatial resolution is quantified using spectral coherence. With each method of calculating wind directions, temporal resolutions on the order of tens of seconds can be achieved. The accuracy of FODS wind directions was evaluated against a sonic anemometer, showing deviations of less than $15^{\circ}$ most of the time. The applicability of FODS for wind direction measurements in different environmental conditions is tested by analyzing the dependence of FODS wind direction accuracy and observable scales on environmental factors. Finally, we demonstrate the potential of this technique by presenting a period that displays spatial and temporal structures in the wind direction.

SIGNIFICANCE STATEMENT: We developed a way to measure wind direction in a spatially and temporally distributed way by distributed temperature sensing, providing an unprecedented concurrent spatial and temporal resolution. Temporally and spatially distributed measurements are necessary in order to be able to better understand spatially heterogeneous processes in the atmosphere. By using optical fibers with attached cone-shaped microstructures, horizontal wind directions can be measured during different environmental conditions. The quality of the measured wind direction is quantified by comparison with sonic anemometer measurements, with deviations between the instruments less than $15^{\circ}$ most of the time. Structures in the wind direction are resolvable down to a time scale of tens of seconds and a spatial scale of meters.
\end{abstract}

KEYWORDS: Atmosphere; Atmosphere-land interaction; Small scale processes; In situ atmospheric observations; Measurements; Spectral analysis/models/distribution; Time series; Field experiments; Regional effects; Wind effects

\section{Introduction}

Exchange mechanisms of momentum, mass, and energy between the surface and the atmosphere in the stable weakwind boundary layer are crucial for our understanding of many processes, including, for example, fog formation (Oke 1987), pollutant dispersal (Hanna 1986, 1990), forest-atmosphere gas exchange (Oliveira et al. 2013; Freundorfer et al. 2019), or frost formation (Whiteman 2000). These exchange processes typically are statistically parameterized using similarity theories (Holtslag and De Bruin 1988). Many of the fundamental assumptions made by similarity theories, however, e.g., spatial homogeneity and Taylor's hypothesis of frozen turbulence (Taylor 1938), are not fulfilled in the surface layer, particularly

\footnotetext{
Denotes content that is immediately available upon publication as open access.

Corresponding author: Anita Freundorfer, anita.freundorfer@ uni-bayreuth.de
}

during nocturnal weak-wind situations and during the occurrence of submesoscale processes (Mahrt et al. 2009; Thomas 2011; Sun et al. 2012, 2015, 2020; Pfister et al. 2021b). The inapplicability of these assumptions hampers climate and weather models since it leads to incorrect predictions of turbulent fluxes at the land surface (Holtslag and De Bruin 1988; Holtslag et al. 2013; Davy and Esau 2014; Lapo et al. 2019). Due to the invalidity of Taylor's hypothesis and the local character of turbulent quantities, spatially and temporally explicit measurements are needed in order to understand the nature of submesoscale processes, building toward the goal of improving model parameterizations (Mahrt et al. 2009; Acevedo et al. 2014; Thomas 2011; Pfister et al. 2021a; Mahrt 2020). Spatially explicit measurements are also useful in other conditions than during a stable weakwind boundary layer, since internal boundary layers and heterogeneous turbulence can occur during strong wind conditions

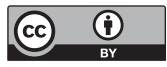

This article is licensed under a Creative Commons Attribution 4.0 license (http://creativecommons.org/ licenses/by/4.0/). 
and in the convective boundary layer in the presence of surface heterogeneities (Mahrt and Vickers 2005; Mott et al. 2017; Fritz et al. 2021).

Distributed temperature sensing (DTS) with fiber-optic cables has been shown to be a promising candidate for fulfilling this research need (Thomas et al. 2012; Peltola et al. 2021). Most often, the Raman scattering method is used for DTS, where some fraction of a light pulse sent into the cable is scattered back at shifted wavelengths (Thomas and Selker 2021). The intensity distribution of the frequency-shifted reflection facilitates calculating the temperature of the optical fiber at the point of the scattering. The location of the scattering event can be obtained from the light pulses time of flight (Selker et al. 2006a,b; Thomas and Selker 2021), so that spatially distributed temperature measurements can be achieved. Apart from measuring temperatures, the DTS technique has also been used successfully for spatially and temporally distributed measurements of other atmospheric variables such as solar radiation (Petrides et al. 2011), wet-bulb temperature (Euser et al. 2014; Schilperoort et al. 2018), and wind speed (Sayde et al. 2015). This broader application of distributed temperature sensing will be called fiber-optic distributed sensing (FODS).

For the observation of wind speeds through FODS, an active approach is used and the fiber is utilized as a spatially resolved hotwire anemometer (van Ramshorst et al. 2020; Sayde et al. 2015). With this approach, temperature and wind speed observations can be obtained at temporal resolutions down to seconds and on spatial scales from tens of centimeters up to kilometers (Pfister et al. 2019). As such, FODS is capable of monitoring turbulent and submesoscale events. It could fill the gap between point measurements with very high temporal resolutions from instruments like sonic anemometers and spatially distributed observations from remote sensing with a coarser resolution.

In order for FODS to be used as a full three-dimensional atmospheric flow sensor, however, it is necessary to not only measure wind speed but also its direction. A fully threedimensional flow sensor would be a valuable tool for understanding nonlocal processes. As an example, it could be used for tracing vertical fluxes of sensible heat or other scalars during the occurrence of advection or cold-air drainage, leading to a better understanding of $\mathrm{CO}_{2}$ sinks and sources.

Lapo et al. (2020b) used microstructures attached to a pair of actively heated fibers in opposing directions in order to introduce directional sensitivity to the FODS measurements. These microstructures artificially generate a convective heat loss that depends on the direction of the flow compared to the pair of fibers. By means of wind tunnel experiments as well as computational fluid dynamics simulations they showed that the usage of microstructures on pairs of actively heated fibers allows for determining the sign of the wind direction for a flow along the axis of the fibers. In order for the microstructure technique to be of use for future field experiments its performance needs to be tested for cases when the flow is not aligned with the fiber axis and in an environmental deployment.

Here we present results from the first field experiment in which the microstructure approach to FODS wind direction was applied in order to obtain spatially distributed measurements of wind directions. Horizontal wind directions are computed solely based on fiber-optic measurements and compared to sonic anemometer data (section 3a, section 4). We quantify the quality of the FODS wind components (section 3c) and analyze the minimal resolvable scales in both space and time domains (section 3b). Furthermore, the dependence of the fiber-derived wind component data on environment conditions such as wind direction steadiness or wind speed is investigated (section 3d). Finally, spatially distributed wind directions are demonstrated for an exemplary period, followed by a discussion on design considerations for future experiments (section 4).

\section{Material and methods}

\section{a. Site and instruments}

The data used for this study were obtained as a part of the Large-Eddy Observatory Voitsumra Experiment (LOVE19) in the period of 15-28 July 2019. During this period a total of $241 \mathrm{~h}$ of FODS data were collected (Lapo et al. 2020a). The experiment was conducted on a grassland site located in the midrange mountain valley "Weißenstädter Becken" at a height of $624 \mathrm{~m}$ MSL (Fig. 1c). The basin is confined by mountains to the north ( $877 \mathrm{~m} \mathrm{MSL})$ and to the south (1051 m MSL), leading to predominantly westerly flows and nightly cold-air pooling.

The fiber-optic cable was a loosely buffered cable containing four $50-\mu \mathrm{m}$ multimode bend-insensitive cores inside a highresistance stainless steel sheath filled with gel (tube inner diameter $=1.06 \mathrm{~mm}$, outer diameter $=1.32 \mathrm{~mm}$, Model C-Tube, Solifos AG, Switzerland, resistance $=1.8 \Omega \mathrm{m}^{-1}$ ). Around the stainless steel sheath there was a $0.2-\mathrm{mm}$ polyethylene (PE) coating for electric insulation. The fiber was mounted in a crosswise design on aluminum trusses. We will refer to this setup of fiber-optic cables as the FODS cross (Fig. 1a). One branch of the cross at a height of $2.27 \mathrm{~m}$ above ground level was oriented to have an azimuth of $\beta=61^{\circ}$ referred to as EW branch from here on according to the approximate orientation, and the second one perpendicular to that with an azimuth of $\beta=151^{\circ}$ referred to as "NS branch" at height of $2.02 \mathrm{~m}$. The offset between the heights of the orthogonal branches prevents any influence of one branch on the other at the intersection. Each branch consisted of four parallel fibers here referred to as the "quartet" which were gently looped around at the ends with a minimum bend radius of $15 \mathrm{~cm}$ to avoid signal loss from sharp bends. On two of the quartet fibers, cones made from PE with a diameter and height of $12 \mathrm{~mm}$, respectively, had been attached by injection molding, with a $2-\mathrm{cm}$ distance between the individual cones. This distance, size, and aspect ratio was determined as optimal by Lapo et al. (2020b). The quartet was arranged such that the two fibers without cones were next to each other horizontally and above the two fibers with cones. The separation of the quartet fibers was $15 \mathrm{~cm}$ horizontally and vertically, respectively (Fig. 1d). The array was part of a 910-m-long section of continuous fiber-optical cable (Lapo et al. 2021). 
a)
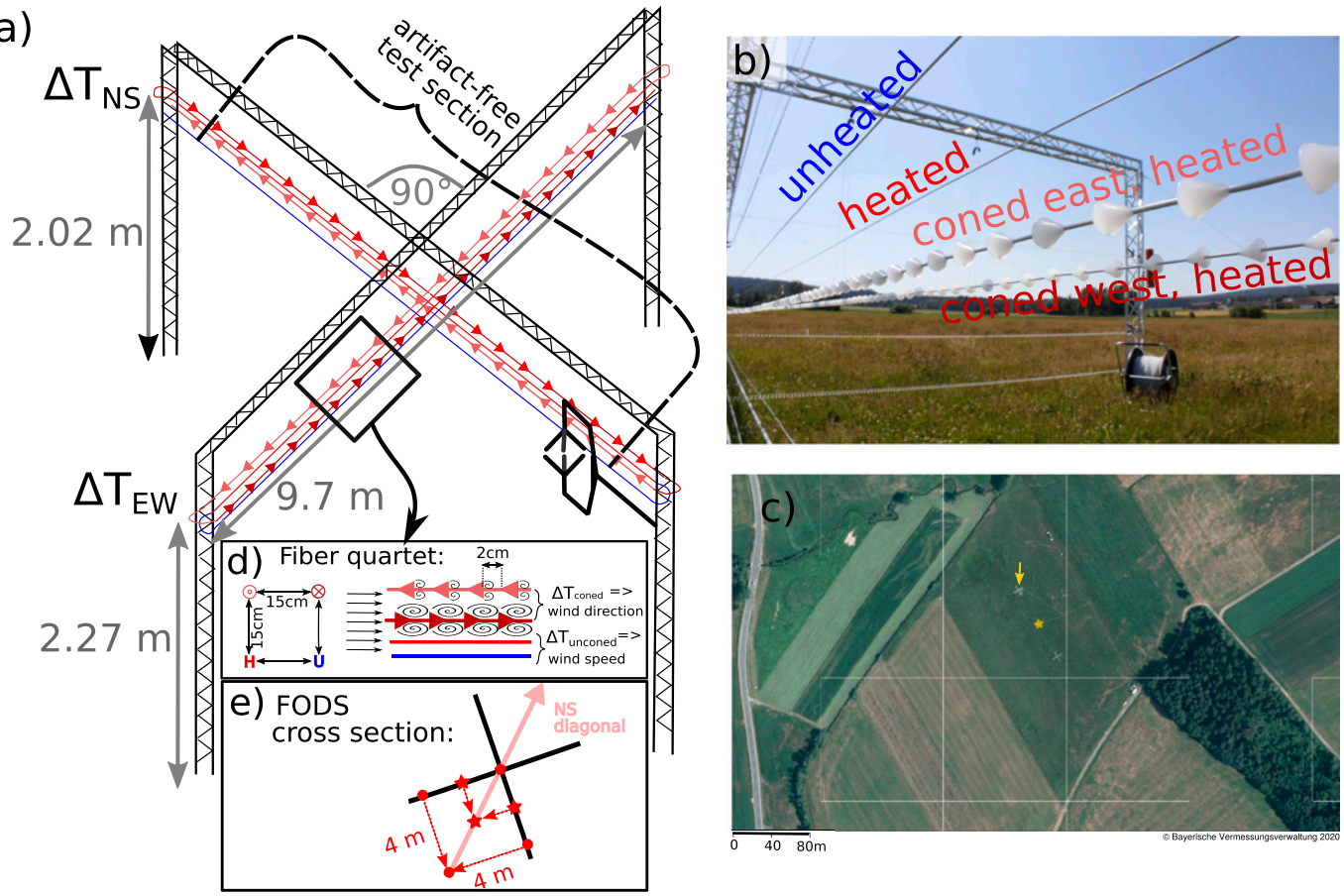

FIG. 1. (a) Schematic of the fiber-optic distributed sensing (FODS) cross showing the crosswise setup of the fiber quartets (one heated and one unheated fiber without cones and two heated fibers with cones pointing in opposing directions). The reference sonic anemometer was attached to the southerly end of the north-south-oriented branch. (b) Photo of the fiber quartet. (c) Image of the experimental site showing instrument the locations. The FODS cross is indicated by an arrow and the tower with the profile of sonic anemometers by a star. (d) Closeup schematic of the fiber quartet. The wind direction can be determined from temperature differences between neighboring coned fibers and the wind speed from temperature differences of neighboring cone-free fibers. (e) Schematic of the cross section demonstrating how data are used from both test sections for obtaining spatially distributed FODS time series.

Both of the fibers with cones and one of the fibers without cones of each branch were heated by applying a constant electric current to the stainless steel sheath using an electrical heating unit (Model Heat Pulse System, Silixa, London, United Kingdom). Temperatures along the fiber were measured by a high-resolution DTS instrument (Model $5 \mathrm{~km}$ Ultima, Silixa) with a spatial sampling resolution of $0.127 \mathrm{~m}$, a physical resolution of $0.3 \mathrm{~m}$, and a temporal resolution of $1 \mathrm{~s}$ in a single-ended configuration.

As reference for the DTS measurements, a sonic anemometer (Model USA-1, Metek GmbH, Elmshorn, Germany) was attached to the southern end of the NS branch at a distance of $0.37 \mathrm{~cm}$ to the closest point of the fiber quartet and $4.07 \mathrm{~m}$ to the center of the cross center. A profile of four sonic anemometers (Model CSAT3, Campbell Scientific Inc., Logan, Utah) on a tower (star symbol in Fig. 1c) at a distance of $39 \mathrm{~m}$ from the FODS structure was used for determining wind regimes (section 3d).

\section{b. DTS data processing}

DTS measurements were done in single ended configuration. Only one out of the four cores of the fiber is used for this analysis. Increasing the length of the measured core past one kilometer would have led to increasing instrument noise due to the smaller signal-to-noise ratio further along the optical path (Lapo et al. 2021), which makes an exact alignment of the test sections between multiple cores challenging. The calibration parameters necessary for converting the measured intensities of the Stokes and antiStokes signal into fiber temperatures were calculated through matrix inversion according to Hausner et al. (2011) using the pyfocs code by Lapo and Freundorfer (2020). For the matrix inversion method, three sections of fiber with a known temperature are necessary. The sections used for the calibration where between 1.2 and $1.5 \mathrm{~m}$ long, which corresponds to 10-12 individual measurement points along the fiber.

Since the water baths conventionally used for this purpose are challenging to keep well mixed, clean, and at a stable temperature in outdoor applications over a long period, we used solid state reference baths for the very first time (Lapo et al. 2021). Each of them consist of a 20-kg block of copper with a duct into which a section of fiber-optic cable can be inserted. The copper block can be either heated or cooled thermoelectrically to a user-selected temperature by Peltier elements and the temperature of the block is monitored by a PT100 resistance thermometer (class A, 4-wire connection). The setup of thermoelectrically temperature-regulated copper 
blocks is stored in a insulated case in order to keep the energy exchange with the environment minimal.

After calibrating the fiber temperatures, the artifact-free regions of the fiber cross are identified while regions potentially contaminated by artifacts from fiber holders (Pfister et al. 2019) are discarded. This results in an 8-m-long test section that is not influenced by any edge effects for each of the branches (Fig. 1a).

\section{c. Deriving wind direction from DTS data}

Once artifact-free sections have been identified, wind speeds and directions can be calculated from parallel strands of fibers. Wind speed is calculated from the two parallel sections of fiber that do not possess cones, one of them heated and one unheated (Fig. 1d) (Sayde et al. 2015; van Ramshorst et al. 2020). Following Sayde et al. (2015), wind speeds are derived using

$u_{n}=\left[\frac{0.5 P \pi^{-1} r^{-1}+\epsilon \sigma\left(T_{\mathrm{unh}}^{4}-T_{\mathrm{h}}^{4}\right)+0.5 c_{p} \rho r\left(\frac{\partial T_{\mathrm{h}}}{\partial t}-\frac{\partial T_{\mathrm{unh}}}{\partial t}\right)}{C(2 r)^{m-1} \operatorname{Pr}_{\mathrm{unh}}\left(\frac{\operatorname{Pr}_{\mathrm{unh}}}{\operatorname{Pr}_{\mathrm{h}}}\right)^{0.25} K_{A} \nu^{-m}\left(T_{\mathrm{h}}-T_{\mathrm{unh}}\right)}\right]^{1 / m}$

The power of the applied heating is $P\left(\mathrm{~J} \mathrm{~s}^{-1} \mathrm{~m}^{-1}\right)$, the cable radius $r(0.00066 \mathrm{~m})$, the Stefan-Boltzmann constant $\sigma$, the fibers' surface emissivity $\epsilon(0.95)$, specific heat capacity $c_{p}\left(1 \mathrm{~J} \mathrm{~kg}^{-1} \mathrm{~K}^{-1}\right)$, density $\rho\left(1000 \mathrm{~kg} \mathrm{~m}^{-3}\right)$ the thermal conductivity $K_{A}\left(\mathrm{~J} \mathrm{~s}^{-1} \mathrm{~m}^{-1} \mathrm{~K}^{-1}\right)$, and kinematic viscosity $\nu$ $\left(\mathrm{m}^{2} \mathrm{~s}^{-1}\right)$ of air; $T_{h}(\mathrm{~K})$ is the temperature of the heated fiber and $T_{\mathrm{unh}}(\mathrm{K})$ the temperature of the unheated fiber, which is assumed to be equal to the air temperature, and $\operatorname{Pr}_{\mathrm{h}}$ and $\mathrm{Pr}_{\mathrm{unh}}$ are the Prandtl numbers at the temperatures $T_{\mathrm{h}}$ and $T_{\text {unh }}$, respectively (Lapo 2021). This is a slightly simplified version of the energy balance equation for calculating wind speeds from fiber temperatures found in Sayde et al. (2015) due to the use of matching fiber types between heated and unheated fiber. The use of matching fiber types removes the necessity to take into account the shortwave radiation for the energy balance as it can be assumed to be identical for two parallel, identical strands of fiber (van Ramshorst et al. 2020). To optimize the wind speeds predicted by the FODS, an hourly effective heating rate is employed, that minimizes the bias between FODS and sonic wind speeds (Lapo et al. 2021). This results in an average absolute deviation of $0.2 \mathrm{~m} \mathrm{~s}^{-1}$ between FODS-derived wind speeds and wind speeds measured by the sonic and a mean signed deviation of $0.001 \mathrm{~m} \mathrm{~s}^{-1}$.

The sensitivity of FODS derived wind speed depends on the angle between the wind vector and the fiber, $\alpha$. Two different versions of accounting for the angle $\alpha$ can be applied. Sayde et al. (2015) calculate the actual wind speed by

$$
u_{\mathrm{DTS}, \mathrm{S}}=\frac{u_{n}}{\sqrt{\sin ^{2} \alpha+k^{2} \cos ^{2} \alpha}} .
$$

For the sensitivity factor $k$ a value of 0.2 was chosen. The FODS setup used in the LOVE19 experiment features two perpendicular branches of test sections (Fig. 1a). Assuming the flow is approximately horizontal, Eq. (2) can be applied to both branches of the cross simultaneously, using $\alpha_{2}=90^{\circ}-\alpha$ as the attack angle of the wind onto the second branch. As a result, the crosswise geometry turns Eq. (2) into an equation system that can be solved for the angle $\alpha$ and the total wind speed $u_{\mathrm{DTS}, \mathrm{S} 15}$ without a priori knowledge about the wind direction, e.g., by a sonic anemometer. The total wind speed is then

$$
u_{\mathrm{DTS}, \mathrm{S} 15}=\sqrt{\frac{u_{\mathrm{EW}}^{2}+u_{\mathrm{NS}}^{2}}{1+k^{2}}}
$$

while the angle between the NS branch and the wind direction is

$$
\alpha_{\mathrm{NS}, \mathrm{S} 15}=\mathbb{R}\left\{\arctan \left(\sqrt{\frac{u_{\mathrm{NS}}^{2}-k^{2} u_{\mathrm{EW}}^{2}}{u_{\mathrm{EW}}^{2}-k^{2} u_{\mathrm{NS}}^{2}}}\right)\right\},
$$

where $u_{\mathrm{NS}}$ is the speed calculated from the north-south (NS)oriented branch using Eq. (1) and $u_{\mathrm{EW}}$ is calculated from the east-west (EW) branch. For cases with flow close to parallel to a fiber, when $u_{\mathrm{EW}}<k u_{\mathrm{NS}}$ or $u_{\mathrm{EW}}<k^{-1} u_{\mathrm{NS}}$, the argument of the square root is negative. In that case, only the real part $R$ of the resulting complex number needs to be considered.

Note that Eq. (4) does not entirely determine the wind direction. Within a horizontal plane, there are four possible flow directions which result in an angle of $\alpha_{\mathrm{NS}, \mathrm{S}}$ with the NS branch, one in each quadrant of the FODS cross. To determine the actual flow direction, the temperature difference between neighboring pairs of coned fibers can be evaluated by

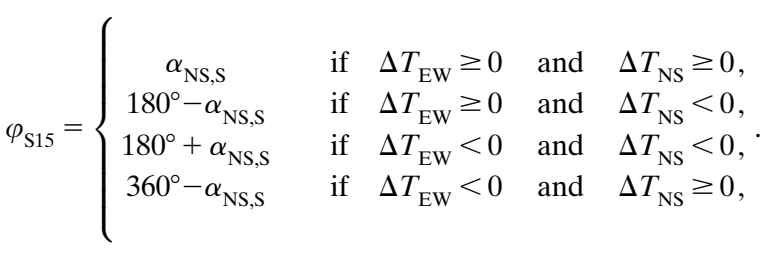

where $\Delta T_{\mathrm{EW}}=T_{\mathrm{Ep}}-T_{\mathrm{Wp}}$ is the temperature difference between the coned fibers on the EW-oriented test section of the FODS cross, with $T_{\mathrm{Wp}}$ being the temperature fiber with cones pointing toward the west and $T_{\mathrm{Ep}}$ the temperature of the fiber with cones pointing toward the east. Similarly, $\Delta T_{\mathrm{NS}}=T_{\mathrm{Np}}-$ $T_{\mathrm{Sp}}$ is the temperature difference between the coned fibers on the EW-oriented test section, $T_{\mathrm{Np}}$ the temperature of the fiber with cones pointing to the north and $T_{\mathrm{sp}}$ the temperature of the fiber with cones pointing to the south. $\varphi_{\mathrm{S} 15}$ is the wind direction in a coordinate system aligned with the FODS cross.

Recently, van Ramshorst et al. (2020) proposed using

$$
u_{\mathrm{DTS}, \mathrm{VR} 20}=\frac{u_{n}}{\sin ^{m_{1}}(\alpha)}
$$

with $m_{1}=1.05$ in order to account for the dependence of the fibers sensitivity to wind speed on the angle between wind direction and fiber axis. Equation (6) can also be solved for the angle between wind direction and NS fiber, yielding

$$
\alpha_{\mathrm{NS}, \mathrm{VR} 20}=\arctan \left[\left(\frac{u_{\mathrm{NS}}}{u_{\mathrm{EW}}}\right)^{2 / m}\right] .
$$


To determine the actual flow direction $\varphi_{\mathrm{VR} 20}$, Eq. (5) can be used again.

Both of those methods rely on the assumption that the flow is approximately horizontal, which can be inaccurate at times (Ovink et al. 2001). We assume that by using an hourly optimal heating rate that diminishes the bias between FODS and sonic wind speeds, this inaccuracy is minimized.

There is a third option on how to calculate wind directions from the data gained with the crosswise FODS geometry. This novel approach we propose here is different than the methods described previously as it is independent of the temperatures measured by cone-free fibers and of the FODS wind speed measurements. Distributed wind directions by this method solely rely on the temperature differences between the coned fibers through

$$
\varphi_{\mathrm{F} 21}=\left\{\begin{array}{cl}
\arctan \left(\frac{\Delta T_{\mathrm{EW}}}{\Delta T_{\mathrm{NS}}}\right) & \text { if } \quad \Delta T_{\mathrm{NS}} \geq 0, \\
180^{\circ}+\arctan \left(\frac{\Delta T_{\mathrm{EW}}}{\Delta T_{\mathrm{NS}}}\right) & \text { if } \quad \Delta T_{\mathrm{NS}}<0 .
\end{array}\right.
$$

Since a vertical velocity component equally applies to all coned fibers, wind directions calculated by this approach should not be affected by the assumption of a purely horizontal flow direction.

In all three approaches, the azimuth of the FODS cross needs to be corrected for, similar to typical anemometry.

\section{d. Deriving spatially distributed wind directions}

For all three approaches of calculating wind directions, data from both branches of the cross are necessary. For any point except for the center of the cross, this implies using measurements from two different locations at some separation distance (Fig. 1e). For the spatially distributed plots, a diagonal cross section is chosen. For each bin along the diagonal, the data from the closest point on the NS and EW branches, respectively, are used for calculating the wind direction, but also for calculating the distributed wind speed. With the branches of the cross being $8 \mathrm{~m}$ long and intersecting in the middle, this approach of calculating spatially distributed data results in a maximum distance of $4 \mathrm{~m}$ between the respective measurement location of the individual components and the corresponding point on the cross section. Although it is not necessary to take into account two orthogonal branches for obtaining meaningful temperature observations, for sake of consistency, temperatures are calculated along the same diagonal cross section by averaging the measurements from the closest point of each branch.

\section{e. Data analysis tools}

As a quantitative measure to evaluate the frequency-dependent correlation between the wind directions measured by the sonic and those measured by FODS, the magnitude-squared spectral coherence will be used (Bendat and Piersol 1986):

$$
C(f)_{x y}=\frac{\left|P_{x y}\right|^{2}}{P_{x x} P_{y y}},
$$

with the power spectra of the individual variables $P_{x x}$ and $P_{y y}$ and their cross-spectrum $P_{x y}$ obtained using Fourier transform.

Estimating the similarity of angular signals such as wind directions offers additional challenges. It is necessary to ensure that a wind directional difference across the angular discontinuity at the north jump (e.g., an angle of $359^{\circ}$ reported by FODS and an angle of $1^{\circ}$ reported by the sonic) is recognized as a small angular difference. To compare wind directions from FODS and sonic without their $2 \pi$ periodic character interfering, wind directions are converted into unit components by $u_{\text {unit }}=\sin (\varphi)$ and $v_{\text {unit }}=\cos (\varphi)$. Coherences are calculated independently for both unit components and then averaged. Analogously, spectra are also calculated from unit components. For both the coherence and the power spectra, Hann windowing is used in order to avoid leakage effects (Tukey 1958; Kanasewich 1975). Significance of the spectral coherence is tested according to Biltoft and Pardyjak (2009). With 241 independent hourly spectra, coherence is statistically significant at a $5 \%$ significance level for $C_{x y} \geq 0.025$.

\section{f. Flow regime identification}

To investigate whether the wind directions can be determined by FODS for different environmental conditions, FODS data quality needs to be assessed during different flow regimes. We expect the applicability of our three tested wind directional methods to be most sensitive to wind speed or turbulence strength and to the constancy of the wind direction. Thus, we analyze the influence of wind regimes and meandering regimes.

Wind regimes are determined by the sonic anemometer located at 1.24-m height on the tower in 10-min intervals. Intervals where the scalar wind speed is greater than $u_{\mathrm{sc}} \geq$ $0.66 \mathrm{~m} \mathrm{~s}^{-1}$ are classified as strong wind, while any interval with a lower wind speed is classified as weak-wind regime. The threshold was determined by a hockey-stick approach following Sun et al. (2012).

Meandering regimes are determined by the sonic anemometer directly at the FODS cross in 2-m height. To set the focus on nonturbulent directional changes, wind speeds are first vector averaged into $1 \mathrm{~min}$ intervals. Periods with slow but strong directional changes are then identified by comparing wind speeds vectorly averaged and scalarly averaged over an hour through the constancy $C=u_{v} / u_{\text {sc }}$. Periods with a constancy less than $C \leq 0.85$ are classified as meandering. This threshold causes intervals with at least one oscillation back and forth by $90^{\circ}$ within an hour to be classified as meandering. With directional changes of $90^{\circ}$ and more being classified as meandering, it can be tested how the FODS cross resolves wind direction shifts by at least one quadrant.

\section{Results}

\section{a. Qualitative assessment of FODS wind directions}

Comparison with wind directions obtained with a sonic anemometer reveals (Fig. 2) that FODS is able to measure wind directions across multiple flow regimes. FODS wind directions were obtained by spatially averaging over the entire cross setup, but with no temporal averaging applied. The chosen 

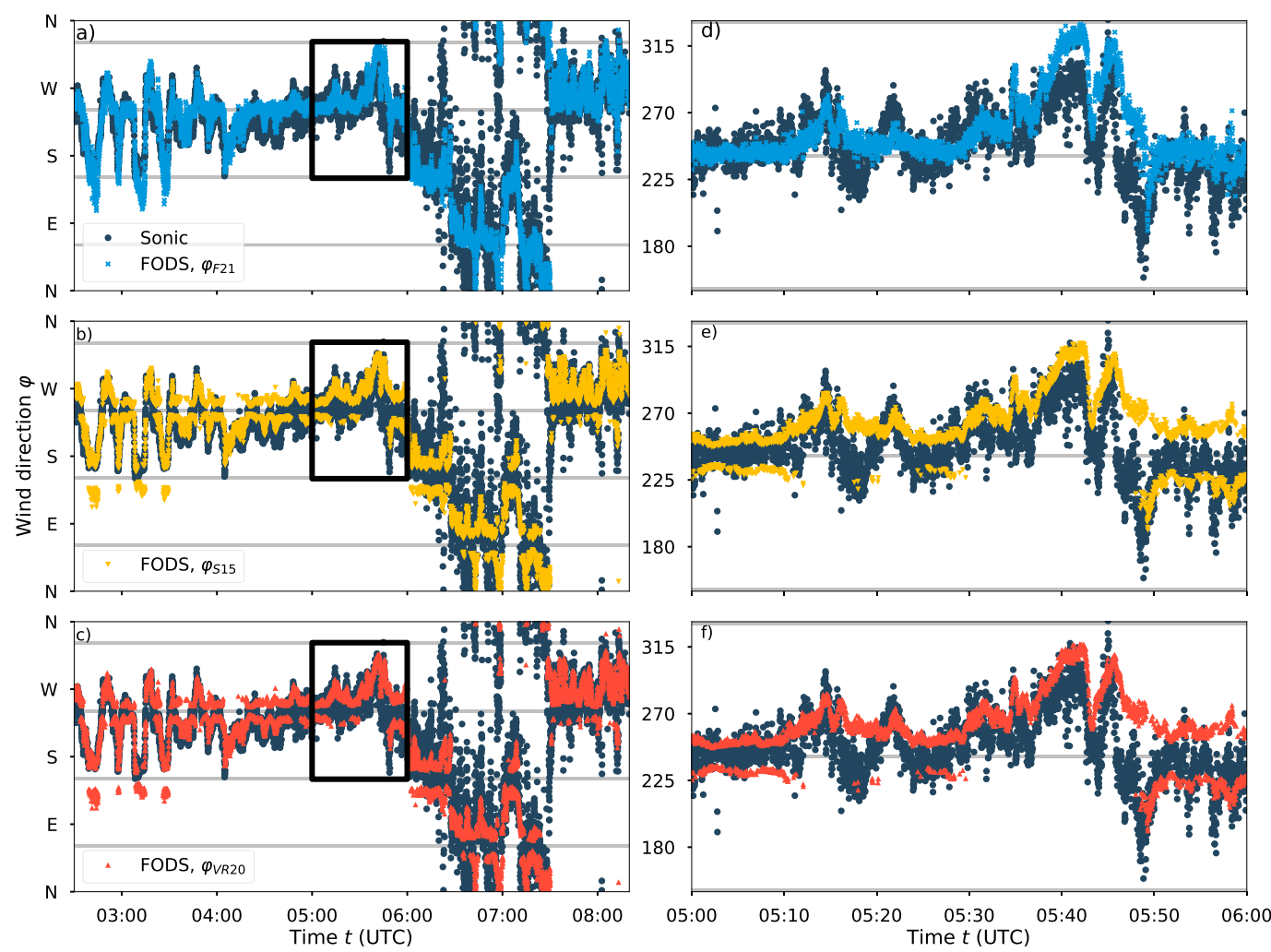

FIG. 2. Time series of wind directions determined by the FODS using (a),(d) the F21 method, (b),(e) the S15 method, and (c),(f) the VR20 method. Black dots represent wind directions measured by the sonic. Horizontal gray lines indicate the directions parallel to the fibers. (d)-(f) Closeup views of the regions highlighted by the black rectangles in (a)-(c).

period features meandering conditions (0230-0400 UTC), the morning transition phase (0400-0600 UTC) (Fritz et al. 2021), a slow wind direction change after sunrise (0600-0700 UTC) followed by higher-frequency turbulent wind direction shifts with a clear mean wind direction during daytime. All methods of calculating FODS wind directions can qualitatively reproduce all of these phenomena during the selected period.

However, with the two methods based on wind speed measurements, S15 and VR20 (Figs. 2b,e and 2c,f), a gap in the FODS wind directions can be identified along the axes of the FODS cross (horizontal gray bars). This lack of points is most evident during the mostly constant westerly wind direction between 0500 and 0600 UTC, during the gradual change of direction between 0600 and 0700 UTC, and during the period with a mostly constant wind direction along the EW branch (0430-0600 UTC). Instead of being aligned with the gray line that indicates the fiber orientation, wind directions calculated via S15 or VR20 alternate above and below once the attack angle of the flow onto the fiber drops below approximately $10^{\circ}$. This behavior is indicative of the wind directions returned by S15 and VR20 methods jumping back and forth between the two adjacent quadrants, suggesting that S15 and VR20 have difficulties correctly sensing directions parallel to any of the test sections. A possible reason for the inability to correctly represent flow parallel to the fiber could be the influence of the binormal velocity component (Ovink et al. 2001). The jumping behavior does not occur for wind direction purely calculated from the coned fibers temperatures, $\varphi_{\mathrm{F} 21}$ (Fig. 2a).

\section{b. Temporal and spatial resolution}

One of the key challenges for aerial DTS deployments is determining the observable scales, since these are inherently related to a variety of transport mechanisms (Thomas et al. 2012). The analysis of minimum resolvable time scale for different averaging lengths in space allows for choosing the optimal combination of time and spatial scale given a specific investigatory focus. Spectral coherence is used in order to estimate on which time scales FODS can measure wind directions (Fig. 3b) and to evaluate the performance of each of the three methods. The frequency at which the coherence drops below $C=0.025$, which corresponds to the $5 \%$ significance level, is used for estimating the minimum resolvable time scale with $\tau=f_{5 \%}^{-1}$. While the power spectra do not give a clear indication of how well the FODS method and observations agree at a given scale, the spectral coherence shows that the similarity between sonic wind directions and FODS-derived wind directions decreases with shorter time scales (Fig. 3). Possible reasons for the decrease of spectral coherence toward shorter time scales include instrument noise, but also a finite reaction time of the fiber due to thermal inertia. When no spatial 

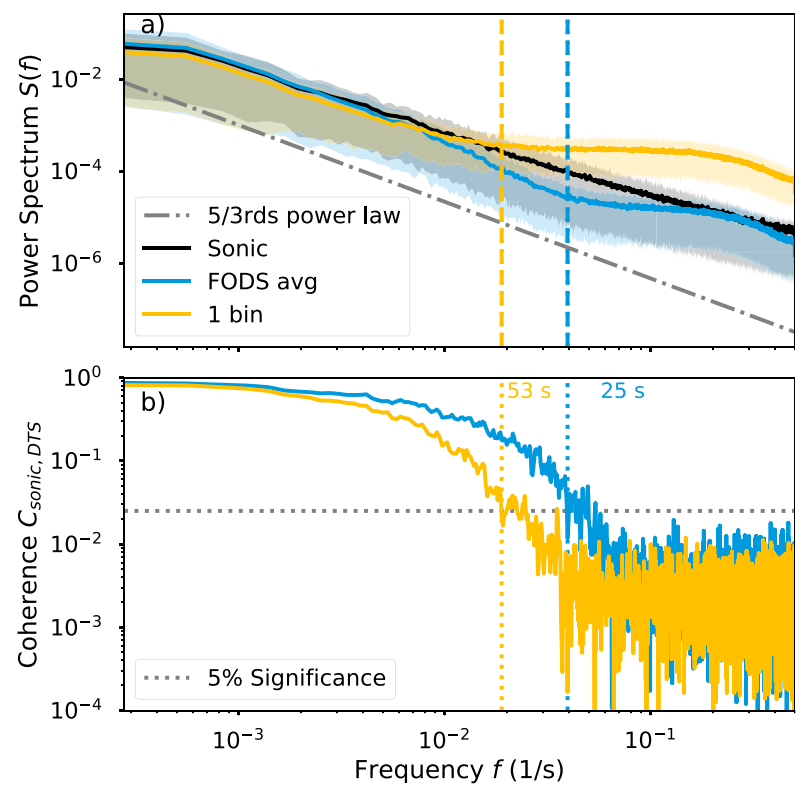

FIG. 3. (a) Average hourly spectra of $u_{\text {unit }}$ and $v_{\text {unit }}$ averaged over $241 \mathrm{~h}$ measured by the sonic (black), by one bin of the FODS cross (orange), and after averaging the unit components $u_{\text {unit, coned }}$ and $v_{\text {unit, coned }}$ over the entire FODS cross (blue). Shaded areas show the region between the 15 th percentile and the 84th percentile of the individual hourly spectra. The dashed gray line represents the $-5 / 3$ power law. (b) Squared spectral coherences between sonic unit components and FODS with one spatial bin (orange) and the entire array (blue). The horizontal dashed line marks the 5\% significance level. Vertical lines indicate the time scale $\tau$ at which the coherence drops underneath the 5\% significance level.

averaging is applied to F21, the minimum resolvable time scale is $\tau_{1 \text { bin }}=53 \mathrm{~s}$. When averaging $\varphi_{\mathrm{F} 21}$ spatially across the entire test setup consisting of the two $8 \mathrm{~m}$ long test sections, the influence of instrument noise gets diminished and the minimum resolvable time scale reduces to $\tau_{8 \mathrm{~m}}=25 \mathrm{~s}$.

Average spectra of the wind direction FODS unit components $u_{\text {unit,coned }}$ and $v_{\text {unit,coned }}$ agree well with those from the sonic for the time scales longer than $\tau$ and also match the spectral slope that is typical for turbulent spectra (Frisch 1995) (Fig. 3a). At shorter time scales the FODS spectra flatten out to a horizontal line as white noise dominates the FODS wind direction. At time scales longer than $\tau_{8 \mathrm{~m}}$ up to around $100 \mathrm{~s}$, the spatially averaged spectra exhibit a drop compared to the sonic spectra. This finding may be explained by some of the structures with time scales shorter than $100 \mathrm{~s}$ being too small to be detected by the complete 8 -m-long branches.

In order find the optimal choice for maximum spatial as well as temporal resolutions, the minimum resolvable time scale according to the $5 \%$ spectral significance test can be identified for all spatial scales between $25.4 \mathrm{~cm}$ (physical resolution of the DTS instrument) and $8 \mathrm{~m}$ (branch length of the cross structure). The dependence of the required temporal averaging length on the size of the spatial average is similar across the different methods of sensing wind directions (Fig. 4). It decreases continuously for increasing spatial averaging up to a

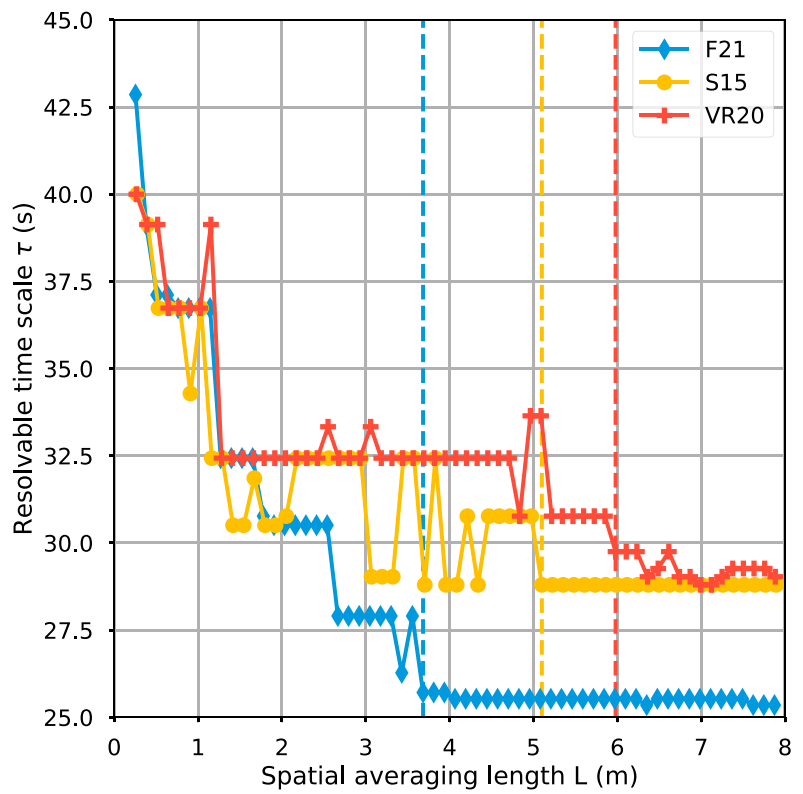

FIG. 4. Dependence of the minimum resolvable time scale on the spatial averaging length for all three methods of detecting wind directions. Dashed vertical lines mark the spatial scale, where further increasing the spatial averaging length leads to little or no improvements in the resolvable time scale.

specific spatial scale, where it reaches a plateau. Once the plateau is reached, extending the spatial averaging length yields marginal improvements in the attainable temporal resolution. The highest temporal resolution can be obtained by our novel F21, which can resolve time scales down to $\tau=26 \mathrm{~s}$ when spatially averaging over $3.6 \mathrm{~m}$. The performance of other two methods is slightly lower with a minimum resolvable time scale of $\tau=29 \mathrm{~s}$ and longer spatial averaging necessary. For $\mathrm{S} 15,5.1 \mathrm{~m}$ of spatial averaging are necessary to reach the plateau, and $6 \mathrm{~m}$ for VR20.

\section{c. Accuracy of FODS wind directions}

To use the distributed wind directions measured by FODS, the measurement accuracy is an important piece of information in addition to the resolvable spatial and temporal scales. Wind directions measured by the sonic anemometer and wind directions measured by FODS are compared at the raw temporal and spatial resolution returned by the DTS device as well as at a resolution that ensures significant coherence between the two devices (Figs. 5a-c). For the temporally and spatially averaged case, a spatial average over the central $3.6 \mathrm{~m}$ of each branch is chosen motivated by the findings of length scale reported above (section $3 \mathrm{~b}$ ). Based on the minimum resolvable time scale for this spatial averaging length (Fig. 4), a running average with a window size of $33 \mathrm{~s}$ is applied to the data. This is the time scale necessary for the VR20 method at the chosen length scale. Although the F21 and the S15 methods are capable of resolving time scales slightly shorter than that, the identical scale of temporal averaging is applied to all three methods to allow comparison of the deviations between them. 

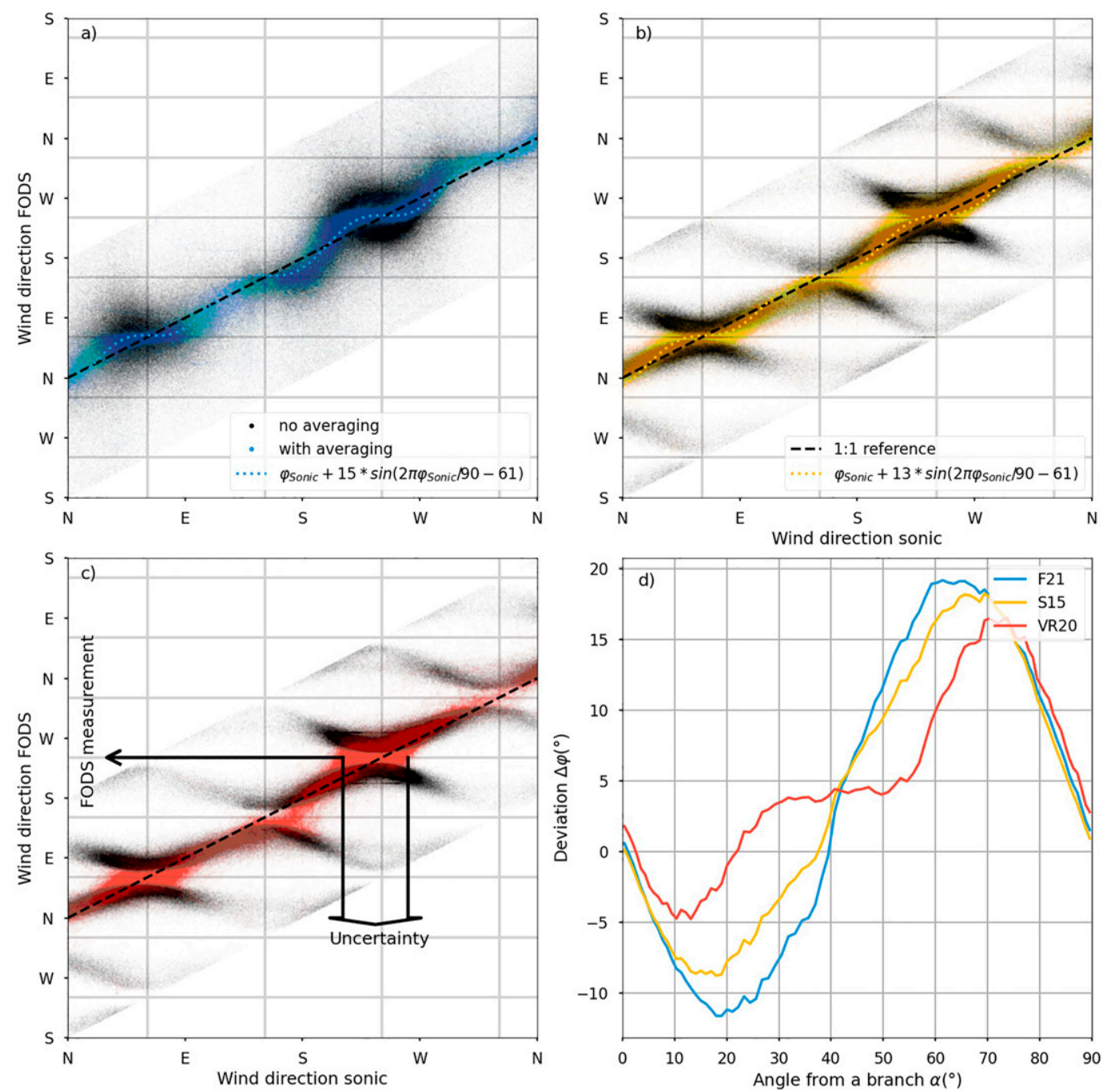

FIG. 5. (a)-(c) Dependence of the wind direction measured by FODS on the sonic wind direction and FODSderived wind direction on the angle between wind and fiber. Black dots represent raw measurements without averaging; colored dots represent measurements at a 3.6-m spatial and 33-s temporal resolutions. Dashed lines are the 1:1 reference lines along which FODS wind directions match the sonic wind directions. Horizontal and vertical gray lines represent the directions along the branches of the FODS cross. (a) Method F21. (b) Method S15. (c) Method VR20. (d) Binned medians of the deviations between FODS wind directions and sonic wind directions for all three methods.

Since the quality of wind speed measurements by FODS depends on the angle of attack between the flow and the fiber (Sayde et al. 2015; van Ramshorst et al. 2020), we likewise expect the deviation between the sonic and FODS derived wind directions to depend on the wind direction. Additionally, the deviations between FODS-detected wind directions and the reference from sonic anemometry were sensitive to the method of choice (Fig. 5). In the case without averaging, methods S15 and VR20 feature a distinct sinusoidal pattern (Figs. 5b,c). The wavelike pattern evolves because at times, although the FODS measurement of the absolute angle between the flow direction and the branches is in close agreement with the sonic measurement, sonic and FODS associate the flow to different quadrants of the FODS cross. Furthermore, gaps at directions parallel to the FODS branches (gray bars in Figs. 5a-c) show that wind directions along FODS branches cannot be detected by S15 and VR20 at the raw resolution, possibly because of the influence of a vertical velocity component (Ovink et al. 2001). Wind direction results by the F21 method at the raw resolution show neither the wavy pattern that is caused by associating the flow to different quadrants, nor gaps at the wind directions parallel to the FODS branches. Instead, F21 measurements at the raw resolution feature a broader scatter around the 1:1 reference line that indicates matching sonic and FODS measurements.

At the 33-s resolution and with averaging over $3.6 \mathrm{~m}$ along the optical cables, the ambiguity in the quadrant determination is reduced and no gap is apparent at the directions parallel to 

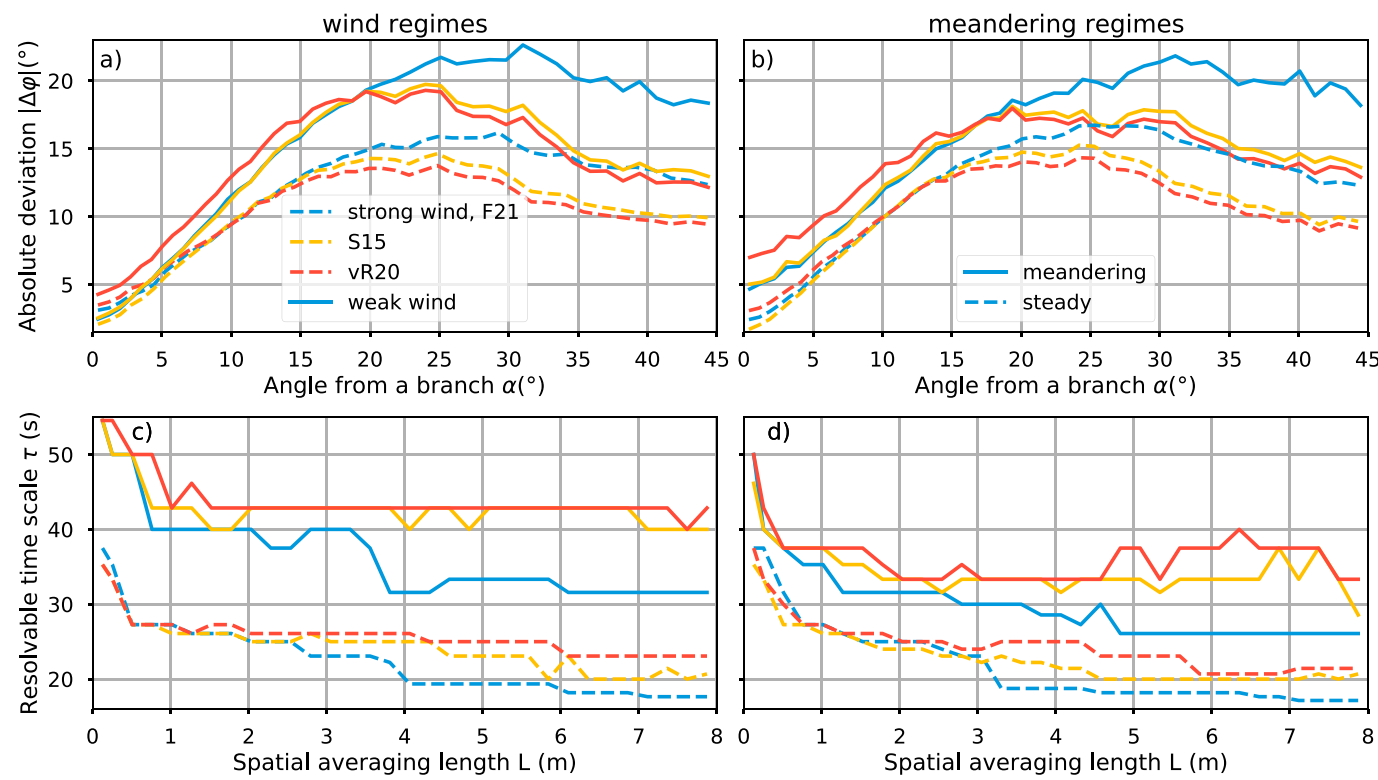

FIG. 6. (a),(b) Deviations between wind directions from the sonic anemometer and from FODS for different regimes. (c),(d) Dependence of the minimum resolvable time scale on the spatial averaging length for the different regimes. (a),(c) Comparison of weak-wind (continuous lines) vs strong-wind (dashed lines) regimes. (b),(d) Comparison of meandering (continuous lines) vs constant (dashed lines) wind direction.

the fibers. Nevertheless, the deviations of the FODS wind directions from the sonic wind directions still exhibit a regular pattern with a $90^{\circ}$ periodicity caused by the angle between the branches of the FODS cross. This pattern is sine shaped in the case of S15 and F21 with an average amplitude of $15^{\circ}$ for F21 and $15^{\circ}$ for S15 according to the results of a nonlinear least squares fit (Figs. 5a,b). VR20 shows a $30^{\circ}$-wide flat region in the middle of each quadrant where the average deviation is less than $5^{\circ}$, framed by regions where the average deviation is up to $15^{\circ}$ (Fig. 5d). The regularity in the pattern of the deviations suggests that by applying a correction term to the FODS wind directions [Eqs. (4), (7), and (8)], the results could be improved further and FODS wind directions could be obtained that match the sonic wind directions more closely. However, for all of the tested methods of calculating the FODS wind directions an uncertainty of around $\pm 15^{\circ}$ will remain for flow close to parallel to the FODS branches (see exemplary arrows in Fig. 5c).

\section{d. Influence of flow regimes on FODS performance}

For FODS wind speeds, Sayde et al. (2015) found a increasing uncertainty in the measurements for increasing wind speed. Hence it is conceivable that also the ability to sense wind directions with FODS depends on environmental conditions. In addition to wind speeds, we also investigated the influence of wind directional changes on the FODS performance. The latter are a common phenomenon in weak-wind boundary layers often termed "meandering" (Anfossi et al. 2005; Mahrt 2007). Meandering is relevant for example for studying dispersion, because it causes standard dispersion models to fail (Anfossi et al. 2006). The general dependence of the absolute deviation between FODS-derived wind directions on the wind direction measured by the sonic stays the same for different wind and steadiness regimes (Fig. 5, Figs. 6a-c). However, the deviations are smaller during strong winds than during weak-wind situations (Fig. 6a) and also smaller during steady wind directions than during meandering (Fig. 6b). While it is intuitive that steady wind directions are easier to represent correctly than meandering, the good performance during strong wind situations is more surprising, given that wind speeds suffered from a larger uncertainty during strong wind situations (Sayde et al. 2015). However, the reduced accuracy during high wind speeds in the case of Sayde et al. (2015) was caused by the temperature difference between heated and unheated cable becoming too small to accurately observe during strong wind speeds. By using a considerably higher heating rate, the problem of too small temperature difference and thus a signal dominated by noise could be avoided during our experiment and situations with strong wind could be captured without problems (Lapo et al. 2021). Whereas vanishing temperature differences between the heated and unheated fiber are not problematic with sufficient heating, very low wind speeds lead to minimized temperature differences between the neighboring coned fibers of opposing direction. When the temperature difference is too small, the signal-to-noise ratio declines and deviations of the FODS wind direction from the sonic wind direction become larger.

Similar to the deviations between FODS and sonic wind directions, the dependence of the minimum resolvable time scales on spatial averaging length also depends on the flow regime. During weak-wind situations, a longer averaging time is necessary for a given spatial averaging length than during strong-wind situations (Fig. 6c). During meandering situations, a longer time 
scale is necessary than during more constant wind directions (Fig. 6d). Since the typical time scales of meandering phenomena are on the order of tens of minutes (Mortarini et al. 2016a,b), the slight prolongation of the necessary averaging time does not hamper the detection and analysis of meandering with FODS.

Note that for any of the regimes, F21 is capable of resolving smaller time scales, but it particularly outperforms the other two methods during weak-wind situations. With the weak signal-to-noise ratio obtained from neighboring coned fibers during weak-wind periods, the FODS wind directions from S15 and VR20 frequently skip back and forth between different quadrants (section 3a, Figs. 2b,c), leading to a comparatively small coherence between the FODS and sonic wind directions and longer averaging required according to the $95 \%$ spectral significance test. With F21, FODS wind directions vary more smoothly, which leads to higher coherence with sonic wind directions down to smaller time scales.

\section{Discussion}

The motivation behind sensing wind directions with FODS is obtaining spatially distributed information about wind directions. With DTS devices being able to measure along kilometers of optical fiber, this could provide us with the means to resolve submesoscale structures, which have scales between tens of centimeters to hundreds of meters, without having the necessity of invoking Taylor's hypothesis to interpret point observations. The ultimate goal is to use FODS as a means for spatially distributed eddy covariance measurements with the aim of analyzing fluxes in a spatial context.

Since F21 is coherent with sonic measurements at smaller length and time scales than the other two methods, this method is used to showcase the observational potential of FODS wind directions using a real-world example. Temporal and spatial averaging is done with a running average according to the results of the coherence analysis (section 3b) for F21. A spatial averaging length of 21 bins (equaling $2.67 \mathrm{~m}$ ) is used, resulting in a required temporal averaging scale of $28 \mathrm{~s}$.

The test setup used for FODS wind directions in our experiment is a $8 \mathrm{~m} \times 8 \mathrm{~m}$ square, which is small compared to the typical size of submesoscale processes (Zeeman et al. 2015). As a result, spatially discrete features were not often resolved within the area covered by the fiber cross. Nevertheless, visual inspection identified periods when the distributed wind directions computed from FODS show a distinct spatial pattern across the observed domain in both space and time. One of the periods featuring a structure in the wind directions occurs between 0442 and 0445 UTC in the morning of 20 July (Fig. 7d). Before that period, the wind direction is southeasterly throughout the observed domain. From 0445 UTC onward, the wind direction is uniformly northeasterly. The shift from a southeasterly through a northerly to a northeasterly wind direction, however, does not happen at once for the entire observed domain, but shows a spatial dependence. A two-dimensional spatial snapshot of wind directions across the FODS cross (Fig. 7b) emphasizes the complex flow patterns that can be resolved even with this small FODS setup.

Though generally very slow, the wind speed exhibits some structure during the selected period (Fig. 7c). The outline of the wind speed structure between 0442 and 0445 UTC shows some similarity to the pattern in the wind direction. The spatially distributed temperatures show slightly lower temperatures at the northern end of the diagonal (Fig. 7a), but no distinct similarities to the wind speed or direction pattern.

The sonic anemometer set up by the southern end of the NS branch confirms the general evolution of wind directions from southeasterly over northerly to northeasterly (Fig. 7e) at its single location. Since only one sonic anemometer was set up by the FODS cross it is impossible to verify the representation of spatial structures from FODS. In fact, the necessity of temperature data from both of the perpendicular branches of the cross for estimating wind directions as well as wind speeds [see Eq. (8)] means that a certain degree of distortion of the observed structure is expected for the geometry of this experiment. However, even if the exact shape of structures might deviate from the one returned by the FODS cross, the results of the coherence analysis suggest, that the spatial and temporal scale of the structures in Fig. 7 can be resolved by FODS, including the smaller blob of northerly wind directions between 0442 and 0443 UTC, which has a spatial scale of approximately $2 \mathrm{~m}$ and an approximate duration of $45 \mathrm{~s}$. Even though a second sonic anemometer within the observational domain would have been useful for validating the spatial structures measured by FODS, none of the currently used measurement instruments in atmospheric sciences is capable of measuring at a sufficient temporal and spatial resolution to match and judge the representation of spatiotemporal structures by FODS, making the observational capability of FODS unique. Consequently, determining meaningful observational scales generally is a major task for the novel FODS method (Peltola et al. 2021; van Ramshorst et al. 2020). Coherence analysis offers a powerful tool which makes it possible to simultaneously estimate spatial and temporal scales, at which structures can be resolved by FODS.

\section{Conclusions}

The ability to measure spatially distributed horizontal wind directions is an important step toward the goal of employing distributed temperature sensing (DTS) as a fully threedimensional, spatially resolved flow sensor for the atmosphere and toward spatially distributed eddy covariance measurements. We demonstrated that it is possible to measure in environmental applications horizontal, spatially distributed wind direction using DTS by employing pairs of actively heated fiber-optic cables with cone-shaped microstructures attached to the fibers in opposing directions. The pairs of fibers were oriented in a crosswise geometry.

We compared three different approaches of calculating wind directions from the DTS data. Two of these approaches are based on two different ways of calculating wind speeds from fiber-optic distributed sensing (FODS) and require pairs of one heated and one unheated fiber in addition to the fibers with microstructures (Sayde et al. 2015; van Ramshorst et al. 2020). The third approach is based purely on measurements by the coned fibers and can be done without the additional cone-free fibers. For all three approaches, resolvable time and spatial scales were evaluated as well as the accuracy of reported wind 


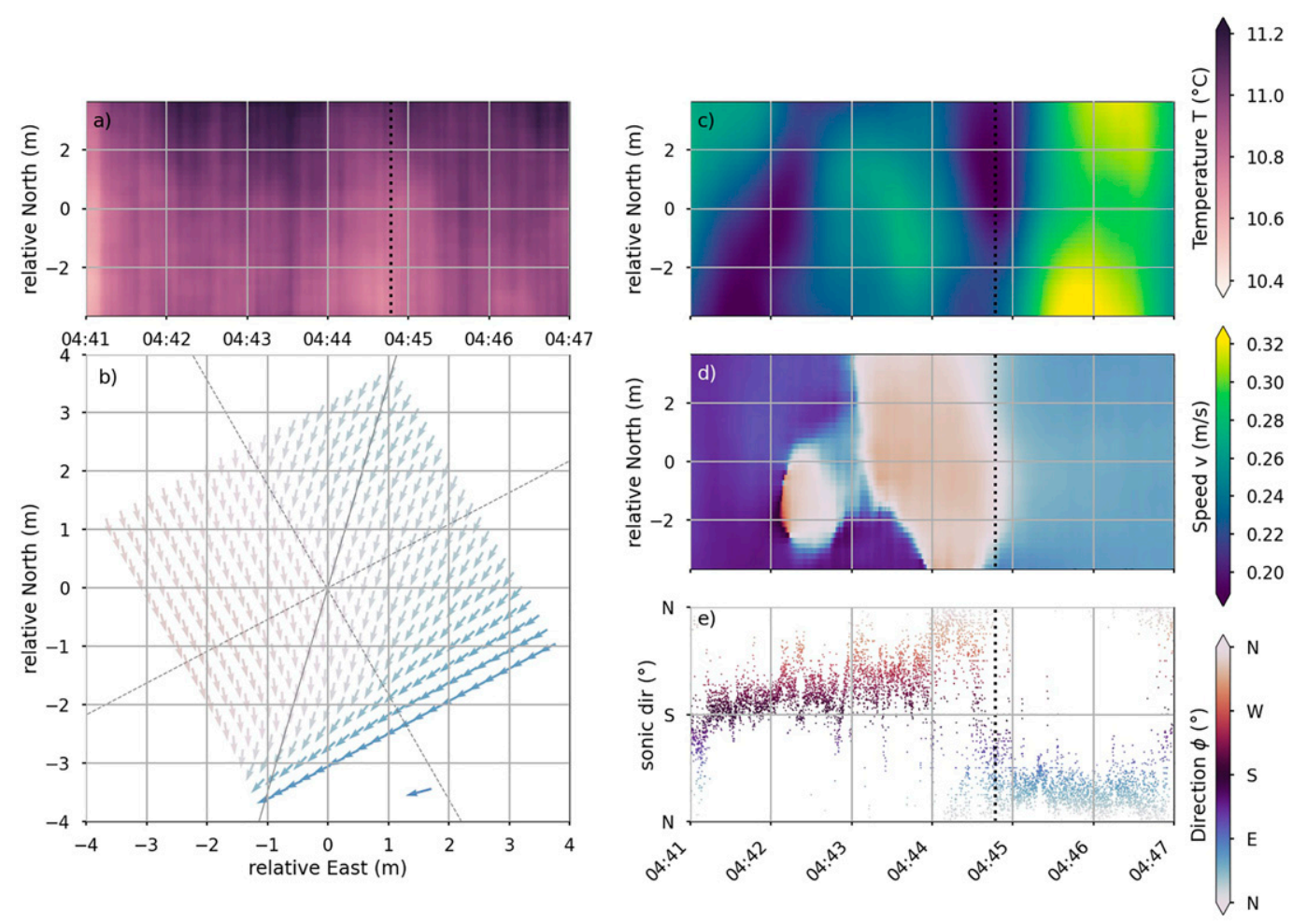

FIG. 7. Spatially distributed measurements by the FODS cross taken on the morning of 20 Jul. (a) Spatially and temporally distributed temperatures along the NS diagonal of the FODS cross. (b) Snapshot of the spatially distributed wind directions taken at 0444:47 UTC. A continuous gray line indicates the diagonal cross section spanned by the temporally distributed plots, while dashed gray lines indicate the location of the optical fibers. The time of the snapshot is indicated by a dashed line in all the temporally distributed plots. An isolated arrow at the bottom right indicates the sonic wind direction at the selected point in time. (c) Spatially and temporally distributed wind speeds along the NS diagonal. (d) Spatially and temporally distributed wind directions along the NS diagonal. (e) Time series of the wind directions measured by the sonic anemometer.

directions. While the accuracy was higher for the wind speedbased approaches during most attack angles, the cone-based method features the highest temporal and spatial resolution out of the three, with a temporal scale of $28 \mathrm{~s}$ accessible at $3-\mathrm{m}$ spatial resolution.

Both accuracy and resolvable scales depend on wind regimes as well as on the wind direction constancy. Particularly during strong wind and without meandering, high temporal and spatial resolutions can be achieved. Highlighting the existence of flow structures with fine spatial and temporal variability, we demonstrated the applicability of FODS for measuring spatially distributed horizontal wind directions.

For future experiments, a larger test setup closer to the anticipated scale of submesoscale phenomena, which ranges from tens of meters to kilometers (Mahrt et al. 2009), would allow for more reliably observing spatial and temporal structures in the wind directions. A geometry of the fiberarray designed with more crossings between fiber quartets would lead to more measurement locations where accurate observations of both wind components can be taken simultaneously. Since a large number of crossings is difficult to obtain while keeping the setup big enough to capture submesoscale processes, a future alternative could be using only a single quartet of fibers for observing the wind direction component along the fiber. This could more easily be applied over large distances. Further research is necessary for developing a method to not only capture the sign, but also the magnitude of the along-fiber wind component with only a single quartet of fibers.

For FODS to be applicable as a full three-dimensional flow sensor, it needs to also measure the vertical wind component. This is more difficult to measure, since the vertical component is much smaller than the horizontal component. Initial testing suggests that the ratio of horizontal to vertical winds removes the directional sensitivity for the vertical component. Thus, future work will focus on making the vertical wind components accessible for FODS.

Acknowledgments. This project has received funding from the European Research Council (ERC) under the European Union's Horizon 2020 research and innovation programme under Grant Agreement 724629, project DarkMix. 
Data availability statement. Data described in this manuscript are accessible through Lapo et al. (2020a) and Freundorfer et al. (2021).

\section{REFERENCES}

Acevedo, O. C., F. D. Costa, P. E. Oliveira, F. S. Puhales, G. A. Degrazia, and D. R. Roberti, 2014: The influence of submeso processes on stable boundary layer similarity relationships. J. Atmos. Sci., 71, 207-225, https://doi.org/10.1175/JAS-D-130131.1.

Anfossi, D., D. Öttl, and G. Degrazia, 2005: An analysis of sonic anemometer observations in low wind speed conditions. Bound.-Layer Meteor., 114, 179-203, https://doi.org/10.1007/ s10546-004-1984-4.

—, S. Alessandrini, S. T. Castelli, E. Ferrero, D. Oettl, and G. Degrazia, 2006: Tracer dispersion simulation in low wind speed conditions with a new $2 \mathrm{D}$ Langevin equation system. Atmos. Environ., 40, 7234-7245, https://doi.org/ 10.1016/j.atmosenv.2006.05.081.

Bendat, J., and A. Piersol, 1986: Random Data. 2nd ed. Wiley Interscience, 566

Biltoft, C. A., and E. R. Pardyjak, 2009: Spectral coherence and the statistical significance of turbulent flux computations. J. Atmos. Oceanic Technol., 26, 403-409, https://doi.org/10.1175/ 2008JTECHA1141.1.

Davy, R., and I. Esau, 2014: Global climate models' bias in surface temperature trends and variability. Environ. Res. Lett., 9, 114024, https://doi.org/10.1088/1748-9326/9/11/114024.

Euser, T., W. M. Luxemburg, C. S. Everson, M. G. Mengistu, A. D. Clulow, and W. G. Bastiaanssen, 2014: A new method to measure Bowen ratios using high-resolution vertical dry and wet bulb temperature profiles. Hydrol. Earth Syst. Sci., 18, 2021-2032, https://doi.org/10.5194/hess-18-2021-2014.

Freundorfer, A., I. Rehberg, B. E. Law, and C. Thomas, 2019: Forest wind regimes and their implications on cross-canopy coupling. Agric. For. Meteor., 279, 107696, https://doi.org/ 10.1016/j.agrformet.2019.107696.

_ 2021: Reference sonic anemometer data for 'Distributed sensing of wind direction using fiber-optic cables.' Zenodo, accessed 25 February 2021, https://doi.org/10.5281/zenodo.4551808.

Frisch, U., 1995: Turbulence: The Legacy of A. N. Kolmogorov. Cambridge University Press, 296 pp., https://doi.org/10.1017/ CBO9781139170666.

Fritz, A., K. Lapo, A. Freundorfer, and C. K. Thomas, 2021: Revealing the evolution and small-scale variability of the morning transition phase in a mountain valley using distributed temperature sensing. Geophys. Res. Lett., 48, e2020GL092238, https://doi.org/10.1029/2020GL092238.

Hanna, S. R., 1986: Spectra of concentration fluctuations: The two time scales of a meandering plume. Atmos. Environ., 20,11311137, https://doi.org/10.1016/0004-6981(86)90145-9.

_ 1990: Lateral dispersion in light-wind stable conditions. Nuovo Cimento, 13C, 889-894, https://doi.org/10.1007/BF02514777.

Hausner, M. B., F. Suárez, K. E. Glander, and N. V. D. Giesen, 2011: Calibrating single-ended fiber-optic Raman spectra distributed temperature sensing data. Sensors, 11, 10 859-10 879, https:// doi.org/10.3390/s111110859.

Holtslag, A. A. M., and H. A. R. De Bruin, 1988: Applied modeling of the nighttime surface energy balance over land. J. Appl. Meteor., 27, 689-704, https://doi.org/10.1175/1520-0450(1988) 027<0689:AMOTNS $>2.0$.CO;2.
— and diurnal cycles: Challenges for weather and climate models. Bull. Amer. Meteor. Soc., 94, 1691-1706, https:// doi.org/10.1175/BAMS-D-11-00187.1.

Kanasewich, E. R., 1975: Time Sequence Analysis in Geophysics. University of Alberta, $352 \mathrm{pp}$.

Lapo, K., 2021: LOVE19 example scripts. Zenodo, https://doi.org/ 10.5281/zenodo.5027181.

— doi.org/10.5281/zenodo.4292491.

_ B. Bijssen, and J. Lundquist, 2019: Evaluation of turbulence stability schemes of land models for stable conditions. J. Geophys. Res. Atmos., 124, 3072-3089, https://doi.org/ 10.1029/2018JD028970.

- A. Freundorfer, A. Fritz, J. Schneider, J. Olesch, W. Babel, and C. K. Thomas, 2020a: Large-Eddy Observatory, Voitsumra Experiment 2019 (LOVE19). Zenodo, accessed 25 February 2021, https://doi.org/10.5281/zenodo.4312976.

,,-- L. Pfister, J. Schneider, J. Selker, and C. Thomas, 2020b: Distributed observations of wind direction using microstructures attached to actively heated fiber-optic cables. Atmos. Meas. Tech., 13, 1563-1573, https://doi.org/10.5194/amt-131563-2020.

,,-- A. Fritz, J. Schneider, J. Olesch, W. Babel, and C. K. Thomas, 2021: The Large-Eddy Observatory Voitsumra Experiment 2019 (LOVE19) with high-resolution, spatiallydistributed observations of air temperature, wind speed, and wind direction from fiber-optic distributed sensing, towers, and ground-based remote sensing. Earth Syst. Sci. Data, https://doi.org/10.5194/essd-2020-392, in press.

Mahrt, L., 2007: Weak-wind mesoscale meandering in the nocturnal boundary layer. Environ. Fluid Mech., 7, 331-347, https:// doi.org/10.1007/s10652-007-9024-9.

_ 2020: Time-space variations of temperature in the nocturnal boundary layer. Quart. J. Roy. Meteor. Soc., 146, 2756-2767, https://doi.org/10.1002/qj.3815.

— scale changes of surface heat flux. Bound.-Layer Meteor., 116, 313-330, https://doi.org/10.1007/s10546-004-1669-z.

— C. K. Thomas, and J. H. Prueger, 2009: Space-time structure of mesoscale motions in the stable. Quart. J. Roy. Meteor. Soc., 75, 67-75, https://doi.org/10.1002/qj.348.

Mortarini, L., S. Maldaner, L. Moor, M. Stefanello, O. Acevedo, G. Degrazia, and D. Anfossi, 2016a: Temperature autocorrelation and spectra functions in low-wind meandering conditions. Quart. J. Roy. Meteor. Soc., 142, 1881-1889, https:// doi.org/10.1002/qj.2796.

—, M. Stefanello, G. Degrazia, D. Roberti, S. T. Castelli, and D. Anfossi, 2016b: Characterization of wind meandering in lowwind-speed conditions. Bound.-Layer Meteor., 161, 165-182, https://doi.org/10.1007/s10546-016-0165-6.

Mott, R., S. Schlögl, L. Dirks, and M. Lehning, 2017: Impact of extreme land surface heterogeneity on micrometeorology over spring snow cover. J. Hydrometeor., 18, 2705-2722, https://doi.org/10.1175/JHM-D-17-0074.1.

Oke, T. R., 1987: Boundary Layer Climates. Routledge, 435 pp.

Oliveira, P. E., O. C. Acevedo, O. L. Moraes, H. R. Zimermann, and C. Teichrieb, 2013: Nocturnal intermittent coupling between the interior of a pine forest and the air above it. Bound.-Layer Meteor., 146, 45-64, https://doi.org/10.1007/ s10546-012-9756-z.

Ovink, R., A. Lamers, A. van Steenhoven, and H. W. M. Hoeijmakers, 2001: A method of correction for the binormal 
velocity fluctuation using the look-up inversion method for hot-wire anemometry. Meas. Sci. Technol., 12, 1208-1213, https://doi.org/10.1088/0957-0233/12/8/330.

Peltola, O., K. Lapo, I. Martinkauppi, E. O’Connor, C. K. Thomas, and T. Vesala, 2021: Suitability of fibre-optic distributed temperature sensing for revealing mixing processes and higherorder moments at the forest-air interface. Atmos. Meas. Tech., 14, 2409-2427, https://doi.org/10.5194/amt-2020-260.

Petrides, A. C., J. Huff, A. Arik, N. V. D. Giesen, A. M. Kennedy, C. K. Thomas, and J. S. Selker, 2011: Shade estimation over streams using distributed temperature sensing. Water Resour. Res., 47, 2-5, https://doi.org/10.1029/2010WR009482.

Pfister, L., K. E. Lapo, C. Sayde, J. Selker, L. Mahrt, and C. K. Thomas, 2019: Classifying the nocturnal atmospheric boundary layer into temperature and flow regimes. Quart. J. Roy. Meteor. Soc., 145, 1515-1534, https://doi.org/10.1002/qj.3508.

-, L. Mahrt, and C. K. Thomas, 2021a: Thermal submeso motions in the nocturnal stable boundary layer. Part 2: Generating mechanisms and implications. Bound.-Layer Meteor., 180, 203-224, https://doi.org/10.1007/s10546-021-00619-z.

$\_, \ldots, \ldots$, and $\_$- 2021b: Thermal submesoscale motions in the nocturnal stable boundary layer. Part 1: Detection and mean statistics. Bound.-Layer Meteor., 180, 187-202, https:// doi.org/10.1007/s10546-021-00618-0.

Sayde, C., C. K. Thomas, J. Wagner, and J. Selker, 2015: Highresolution wind speed measurements using actively heated fiber optics. Geophys. Res. Lett., 42, $10064-10073$, https:// doi.org/10.1002/2015GL066729.

Schilperoort, B., M. Coenders-Gerrits, W. Luxemburg, C. Jiménez Rodríguez, C. Cisneros Vaca, and H. Savenije, 2018: Using distributed temperature sensing for Bowen ratio evaporation measurements. Hydrol. Earth Syst. Sci., 22, 819-830, https:// doi.org/10.5194/hess-22-819-2018.

Selker, J., N. van de Giesen, M. Westhoff, W. Luxemburg, and M. B. Parlange, 2006a: Fiber optics opens window on stream dynamics. Geophys. Res. Lett., 33, L24401, https://doi.org/ 10.1029/2006GL027979.

_ _ and Coauthors, 2006b: Distributed fiber-optic temperature sensing for hydrologic systems. Water Resour. Res., 42, W12202, https://doi.org/10.1029/2006WR005326.
Sun, J., L. Mahrt, R. M. Banta, and Y. L. Pichugina, 2012: Turbulence regimes and turbulence intermittency in the stable boundary layer during CASES-99. J. Atmos. Sci., 69, 338-351, https://doi.org/10.1175/JAS-D-11-082.1.

tions in the stable atmospheric boundary layer. Rev. Geophys., 53, 956-993, https://doi.org/10.1002/2015RG000487.

_- E. E. Takle, and O. C. Acevedo, 2020: Understanding physical processes represented by the Monin-Obukhov bulk formula for momentum transfer. Bound.-Layer Meteor., 177, 69-95, https://doi.org/10.1007/s10546-02000546-5.

Taylor, G. I., 1938: The spectrum of turbulence. Proc. Roy. Soc., 164A, 476-490, https://doi.org/10.1098/rspa.1938.0032.

Thomas, C. K., 2011: Variability of sub-canopy flow, temperature, and horizontal advection in moderately complex terrain. Bound.-Layer Meteor., 139, 61-81, https://doi.org/10.1007/ s10546-010-9578-9.

_, and J. Selker, 2021: Optical fiber-based distributed sensing methods. Springer Handbook of Atmospheric Measurements, T. Foken, Ed., Springer, 609-631.

—, A. M. Kennedy, J. S. Selker, A. Moretti, M. H. Schroth, A. R. Smoot, N. B. Tufillaro, and M. J. Zeeman, 2012: High-resolution fibre-optic temperature sensing: A new tool to study the two-dimensional structure of atmospheric surfacelayer flow. Bound.-Layer Meteor., 142, 177-192, https://doi.org/ 10.1007/s10546-011-9672-7.

Tukey, J. W., 1958: The Measurement of Power Spectra: From the Point of View of Communications Engineering. Dover, 190 pp.

van Ramshorst, J. G., and Coauthors, 2020: Revisiting wind speed measurements using actively heated fiber optics: A wind tunnel study. Atmos. Meas. Tech., 13, 5423-5439, https://doi.org/ 10.5194/amt-13-5423-2020.

Whiteman, C. D., 2000: Mountain Meteorology: Fundamentals and Applications. Oxford University Press, $355 \mathrm{pp}$.

Zeeman, M. J., J. S. Selker, and C. K. Thomas, 2015: Nearsurface motion in the nocturnal, stable boundary layer observed with fibre-optic distributed temperature sensing. Bound.-Layer Meteor., 154, 189-205, https://doi.org/10.1007/ s10546-014-9972-9. 